Discussion Paper No. 925

\title{
CHARACTERIZING MINIMAL IMPARTIAL RULES FOR AWARDING PRIZES
}

\author{
Shohei Tamura \\ The $17^{\text {th }}$ ISER-Moriguchi Prize (2014) \\ Awarded Paper
}

\begin{abstract}
January 2015
The Institute of Social and Economic Research

Osaka University

6-1 Mihogaoka, Ibaraki, Osaka 567-0047, Japan
\end{abstract}




\title{
Characterizing minimal impartial rules for awarding prizes*
}

\author{
Shohei Tamura ${ }^{\dagger}$ \\ January 22, 2015
}

\begin{abstract}
We study the problem of choosing prize winners from among a group of experts when each expert nominates another expert for the prize. A nomination rule determines the set of winners on the basis of the profile of nominations; the rule is impartial if one's nomination never influences one's own chance of winning the prize. In this paper, we consider impartial, anonymous, symmetric, and monotonic nomination rules and characterize the set of all minimal such ones. We show that the set consists of exactly one nomination rule: a natural variant of the plurality correspondence called plurality with runners-up.
\end{abstract}

Keywords: Institutional design; Award rule; Conflict of interest; Impartiality; Plurality correspondence

JEL Classification: D63, D71

${ }^{*}$ The author is grateful to Shinji Ohseto for his helpful suggestions. He also thanks Kazuhiko Hashimoto, Ryo Horii, Shigemi Izumida, Takashi Kunimoto, Mitsunobu Miyake, Shigehiro Serizawa, Koichi Tadenuma, Takashi Ui, Zhen Zhao, and seminar participants at Hitotsubashi University, Osaka University and Tohoku University for their useful comments. An earlier version of this paper entitled "A characterization of minimal impartial rules for awarding prizes" was awarded the 17th Moriguchi Prize by the Institute of Social and Economic Research at Osaka University.

${ }^{\dagger}$ Graduate School of Economics and Management, Tohoku University, Sendai, Miyagi 980-8576, Japan. E-mail address: tamura.econ.tohoku@gmail.com 


\section{Introduction}

Suppose that a foundation is considering awarding a prize to one or more members of a group of experts whose activities advance the public interest. The foundation's leader wishes to select people who truly deserve the prize, but he cannot do so by himself because he lacks the expertise needed to evaluate their merits. Given that situation, this paper considers the design of award rules that base the selection of winners on experts' views. In particular, we study nomination rules that ask each expert to nominate one other expert for the prize; the set of winners is then determined based on the profile of nominations. The challenge of this approach is that certain nomination rules might create conflicts of interest among "selfish" experts. In particular, a person caring only about her own winning might corrupt her nomination if there is a chance that she can influence her own likelihood of receiving the prize. We are thus interested in nomination rules that create no such conflict of interest, and study those satisfying an axiom called impartiality. Impartiality requires that whether one wins the prize should be independent of one's nomination; a selfish person thus has no chance to influence her own winning whenever the rule is impartial.

The aim of this paper is to identify reasonable impartial nomination rules among those satisfying three axioms: anonymity, symmetry, and monotonicity. Anonymity requires that an exchange of nominations between two people should not affect any other person's winning. Symmetry requires the determination of the set of winners to be independent of the indexes of people. Monotonicity requires any subset of winners to be included in the new set of winners when they each obtain an additional nomination from another person.

Now, consider the nomination rule under which all people are always chosen as winners. Although satisfying the three axioms and being impartial, we cannot describe such a nomination rule as reasonable. By always selecting too many winners, without examining their qualifications, it might degrade the prestige of the prize, which the foundation aims to maintain. It might also undermine the social practice of competition. These arguments confirm that it is desirable for a nomination rule to select winners as strictly as possible, leading us to the question of which nomination rules are optimal in this sense subject to all the four axioms.

In this paper, we obtain an explicit answer to that question by exploring minimal nomination rules among those satisfying the four axioms. We define a nomination rule satisfying the four axioms as "minimal" if one cannot make a further refinement to the nomination rule while still preserving the four axioms, that is, if no other nomination rule satisfies the four axioms while assigning to every profile of nominations a set of winners that is smaller, measured by inclusion, than that assigned by the nomination rule under consideration. The result will thus characterize the set of all minimal nomination rules satisfying the four axioms. We show that plurality with runners-up (Tamura and Ohseto, 2014) is the only minimal nomination rule satisfying impartiality, anonymity, symmetry, 
and monotonicity. Plurality with runners-up is a natural variant of the ordinary plurality correspondence. Indeed, the set of winners is always that of plurality winners except when there is a sole plurality winner who defeats the runners-up by only one point; in that case, a runner-up also wins if she nominates the sole plurality winner.

This paper is the first, to our knowledge, to establish a characterization result in the context of impartial nomination rules. Holzman and Moulin (2013) begin this area of study with "single-valued" nomination rules and propose interesting impartial rules dubbed partition methods. Instead of characterizing these partition methods, they establish two impossibility results regarding single-valued impartial nomination rules; one of these states that no such rule simultaneously satisfies two axioms that they call positive unanimity and negative unanimity. ${ }^{1}$ Tamura and Ohseto (2014) then allow rules to be "multi-valued," as is done in our paper, and focus on the discussion of whether Holzman and Moulin's impossibility results hold in a more general class of multi-valued nomination rules. By constructing the "plurality with runners-up" correspondence, they show the existence of an impartial rule meeting positive and negative unanimity when at least four people are involved.

In the closely related context of "impartial division rules," a characterization result has already been established. de Clippel et al. (2008) study the problem of dividing a surplus among a group of partners when each partner represents her subjective opinion about the relative contributions of the others to the surplus. A division rule determines the division of the surplus on the basis of the profile of opinions, and impartiality requires the share of the surplus each person receives to be independent of her opinion. Among four or more partners, the authors propose an infinite family of impartial division rules that aggregate the opinions of all partners in a highly natural way. They then characterize that family by employing several reasonable axioms. A clear difference exists between de Clippel et al.'s result and ours: they characterize the whole class of rules meeting their axioms, whereas we characterize only the minimal rules satisfying our axioms. Nevertheless, this difference does not degrade the importance of our result; as explained above, in our context, the investigation of minimal nomination rules is itself meaningful.

The rest of the paper is organized as follows. In Section 2, we introduce the model and axioms. In Section 3, we state and prove the result. In Section 4, we offer concluding remarks. Some proofs are gathered in Appendix.

\section{Model and Axioms}

Let $N=\{1, \ldots, n\}(n \geq 3)$ be the set of people. For each $i \in N$, let $x_{i} \in N \backslash\{i\}$ denote person $i$ 's nomination. If $x_{i}=j$, it means that $i$ nominates $j$. A list $x=\left(x_{i}\right)_{i \in N}$ is called a

\footnotetext{
${ }^{1}$ Positive unanimity says that a person should (uniquely) win if she is nominated by everybody else. Negative unanimity says that a person should not win if she is not nominated by anybody.
} 
nomination profile. Let $N_{-}^{N}$ denote the set of all nomination profiles. For each $x \in N_{-}^{N}$ and each $i_{1}, \ldots, i_{m} \in N$, where $m=1, \ldots, n$, we sometimes write $x$ for $\left(x_{\left\{i_{1}, \ldots, i_{m}\right\}}, x_{N \backslash\left\{i_{1}, \ldots, i_{m}\right\}}\right)$ for distinguishing the nominations of persons $i_{1}, \ldots, i_{m}$ from those of the others in $x$. For simplicity of notation, we often use $\left(x_{i}, x_{-i}\right)$ instead of $\left(x_{\{i\}}, x_{N \backslash\{i\}}\right)$. A nomination rule is a correspondence $\varphi: N_{-}^{N} \rightarrow 2^{N} \backslash\{\emptyset\}$ that assigns a non-empty subset of people, which we mention as the set of winners, to each nomination profile.

We next introduce four axioms that we impose on nomination rules. First, as our central axiom, impartiality says that each person's winning should be independent of her nomination.

Impartiality: for all $x \in N_{-}^{N}$, all $i \in N$, and all $x_{i}^{\prime} \in N \backslash\{i\}$,

$$
i \in \varphi\left(x_{i}, x_{-i}\right) \Leftrightarrow i \in \varphi\left(x_{i}^{\prime}, x_{-i}\right) \text {. }
$$

Second, we introduce an axiom of anonymity that ensures people to be treated equally as "voters." Suppose that two people, say, $j, k$, exchange their nominations each other. Anonymity says that this exchange should not affect the winning of any other person, say, $i$, so that $j$ and $k$ have the same influence on $i$ 's winning. ${ }^{2}$

Anonymity: for all $x \in N_{-}^{N}$, all $i \in N$, all $j, k \in N \backslash\{i\}$, all $x_{j}^{\prime} \in N \backslash\{j\}$, and all $x_{k}^{\prime} \in N \backslash\{k\}$,

$$
\begin{aligned}
& \text { if } x_{j}^{\prime}=x_{k} \neq j, \text { and } x_{k}^{\prime}=x_{j} \neq k, \\
& \text { then } i \in \varphi\left(x_{\{j, k\}}, x_{N \backslash\{j, k\}}\right) \Leftrightarrow i \in \varphi\left(x_{\{j, k\}}^{\prime}, x_{N \backslash\{j, k\}}\right) .
\end{aligned}
$$

Third, we introduce an axiom of symmetry that ensures people to be treated symmetrically as "candidates." 3 Let $\pi: N \rightarrow N$ be a permutation of $N$. The set of all such permutations is denoted by $\Pi_{N}$. For any $\pi \in \Pi_{N}$ and any $x \in N_{-}^{N}$, let $x^{\pi}$ denote the nomination profile such that $x_{i}^{\pi}=\pi\left(x_{\pi^{-1}(i)}\right)$ for all $i \in N$. Note that $x_{\pi(i)}^{\pi}=\pi\left(x_{i}\right)$ for any $i \in N$, which describes how $\pi$ transforms $x$ into $x^{\pi}$ : if $i$ nominates $j$ in $x, \pi(i)$ nominates $\pi(j)$ in $x^{\pi}$. Symmetry says that $\pi(i)$ should be one of the winners in $x^{\pi}$ whenever $i$ is in $x$.

Symmetry: for all $\pi \in \Pi_{N}$, all $x \in N_{-}^{N}$, and all $i \in N$,

$$
i \in \varphi(x) \Leftrightarrow \pi(i) \in \varphi\left(x^{\pi}\right)
$$

\footnotetext{
${ }^{2}$ Anonymity is then weaker than the axiom called anonymous ballots, requiring that a nomination rule should depend only on the number of nominations each person receives. For consequences of anonymous ballots for impartial nomination rules, see Holzman and Moulin (2013) and Tamura and Ohseto (2014).

${ }^{3}$ Our formulation is done in reference to Holzman and Moulin (2013), who define symmetry for randomized single-valued nomination rules, i.e., functions that assign to each nomination profile the winning probabilities of persons.
} 
Finally, we introduce an axiom of monotonicity that imposes a certain consistency requirement on nomination rules. Monotonicity says that any subset of winners should be included in the new set of winners when they each obtain an additional nomination from another person.

Monotonicity: for all $x \in N_{-}^{N}$, all $i_{1}, \ldots, i_{m} \in N$, all $j_{1}, \ldots, j_{m} \in N$, all $x_{j_{1}}^{\prime} \in N \backslash$ $\left\{j_{1}\right\}, \ldots$, and all $x_{j_{m}}^{\prime} \in N \backslash\left\{j_{m}\right\}$

$$
\text { if } \begin{aligned}
& \left\{i_{1}, \ldots, i_{m}\right\} \subset \varphi(x), \\
& x_{j_{1}}, \ldots, x_{j_{m}} \notin\left\{i_{1}, \ldots, i_{m}\right\}, \text { and } \\
& x_{j_{1}}^{\prime}=i_{1}, \ldots, x_{j_{m}}^{\prime}=i_{m}, \\
\text { then } & \left\{i_{1}, \ldots, i_{m}\right\} \subset \varphi\left(x_{\left\{j_{1}, \ldots, j_{m}\right\}}^{\prime}, x_{N \backslash\left\{j_{1}, \ldots, j_{m}\right\}}\right) .
\end{aligned}
$$

Let $\Phi$ denote the set of all nomination rules satisfying impartiality, anonymity, symmetry, and monotonicity. We say that a nomination rule $\varphi \in \Phi$ is minimal if there is no $\varphi^{\prime} \in \Phi$ such that $\varphi^{\prime} \neq \varphi$ and $\varphi^{\prime}(x) \subset \varphi(x)$ for all $x \in N_{-}^{N}$.

\section{Characterization result}

In this section, we show that plurality with runners-up (Tamura and Ohseto, 2014) is the only minimal nomination rule that belongs to $\Phi$. To introduce the definition of plurality with runners-up, we give some additional notations. Given $x \in N_{-}^{N}$ and $i \in N$, let $s_{i}(x)=\left|\left\{j \in N \backslash\{i\}: x_{j}=i\right\}\right|$ denote person $i$ 's score in $x$. Given $x \in N_{-}^{N}$, let $s_{F}(x)=$ $\max _{i \in N} s_{i}(x)$ and $F_{x}=\left\{i \in N: s_{i}(x)=s_{F}(x)\right\}$ denote the (first) highest score and the set of people obtaining that score in $x$, respectively. Similarly, let $s_{S}(x)=\max _{i \in N \backslash F_{x}} s_{i}(x)$ and $S_{x}=\left\{i \in N: s_{i}(x)=s_{S}(x)\right\}$ denote the second highest score and the set of people obtaining that score in $x$, respectively.

Definition 1 (Tamura and Ohseto, 2014). A nomination rule $\varphi^{*}$ is plurality with runnersup if, for all $x \in N_{-}^{N}$,

(a) if $\left|F_{x}\right|=1$ and $s_{F}(x)-s_{S}(x)=1$, then $\varphi^{*}(x)=F_{x} \cup\left\{i \in S_{x}: x_{i} \in F_{x}\right\}$;

(b) else, $\varphi^{*}(x)=F_{x}$.

In words, plurality with runners-up is the nomination rule such that, not only do the plurality winners always win, but also a runner-up wins if she nominates the sole plurality winner who defeats the runner-up by only one point.

Before we state and prove the result, it should be noted that plurality with runners-up $\varphi^{*}$ is not the unique nomination rule that belongs to $\Phi$. Indeed, for instance, the indifference rule, defined by $\varphi^{i n d}(x)=N$ for all $x \in N_{-}^{N}$, also satisfies impartiality, anonymity, 
symmetry, and monotonicity. But we have $\varphi^{*}(x) \subset \varphi^{i n d}(x)$ for all $x \in N_{-}^{N}$, which is consistent with the claim that $\varphi^{*}$ is the only minimal nomination rule that belongs to $\Phi$.

We show that plurality with runners-up is the only minimal nomination rule satisfying impartiality, anonymity, symmetry, and monotonicity.

Theorem 1. Plurality with runners-up is the only minimal nomination rule satisfying impartiality, anonymity, symmetry, and monotonicity.

Proof. Let $\varphi^{*}$ be the plurality with runners-up as in Definition 1. First of all, we must verify that $\varphi^{*}$ actually satisfies each of the four axioms.

Impartiality Here we propose an alternative verification that would be more intuitive than the one established in Tamura and Ohseto (2014). We note that, for all $x \in N_{-}^{N}$ and all $i \in N$, we have $i \in \varphi^{*}(x) \Leftrightarrow i \in F_{x_{-i}}$, where $F_{x_{-i}}$ denotes the set of people obtaining the first highest score in $x$ provided that $i$ 's nomination is not counted. ${ }^{4}$ Hence, since $F_{x_{-i}}$ is independent of $i$ 's nomination, $\varphi^{*}$ satisfies impartiality.

Anonymity For all $x \in N_{-}^{N}$, all $i \in N$, all $j, k \in N \backslash\{i\}$, all $x_{j}^{\prime} \in N \backslash\{j\}$, and all $x_{k}^{\prime} \in N \backslash\{k\}$, suppose that $i \in \varphi^{*}(x), x_{j}^{\prime}=x_{k} \neq j$, and $x_{k}^{\prime}=x_{j} \neq k$. Let $x^{\prime}=\left(x_{\{j, k\}}^{\prime}, x_{N \backslash\{j, k\}}\right)$. If $i \in F_{x}$, then $i \in F_{x^{\prime}}$, and thus $i \in \varphi^{*}\left(x^{\prime}\right)$. If $i \in S_{x}$, we have $\left|F_{x}\right|=1, s_{F}(x)-s_{S}(x)=1$, and $x_{i} \in F_{x}$. Then, we have $i \in S_{x^{\prime}},\left|F_{x^{\prime}}\right|=1, s_{F}\left(x^{\prime}\right)-s_{S}\left(x^{\prime}\right)=1$, and $x_{i}^{\prime}=x_{i} \in F_{x^{\prime}}$. Therefore, we obtain $i \in \varphi^{*}\left(x^{\prime}\right)$.

Symmetry For all $\pi \in \Pi_{N}$, all $x \in N_{-}^{N}$, and all $i \in N$, suppose that $i \in \varphi^{*}(x)$. Note that $s_{\pi(j)}\left(x^{\pi}\right)=s_{j}(x)$ for any $j \in N$. Therefore, if $i \in F_{x}$, we have $\pi(i) \in F_{x^{\pi}}$, and thus $\pi(i) \in \varphi^{*}\left(x^{\pi}\right)$. If $i \in S_{x}$, we have $\left|F_{x}\right|=1, s_{F}(x)-s_{S}(x)=1$, and $x_{i} \in F_{x}$, implying that $\pi(i) \in S_{x^{\pi}},\left|F_{x^{\pi}}\right|=1, s_{F}\left(x^{\pi}\right)-s_{S}\left(x^{\pi}\right)=1$, and $x_{\pi(i)}^{\pi}=\pi\left(x_{i}\right) \in F_{x^{\pi}}$. Hence, we obtain $\pi(i) \in \varphi^{*}\left(x^{\pi}\right)$.

Monotonicity For all $x \in N_{-}^{N}$, all $i_{1}, \ldots, i_{m} \in N$, all $j_{1}, \ldots, j_{m} \in N$, all $x_{j_{1}}^{\prime} \in N \backslash\left\{j_{1}\right\}, \ldots$, and all $x_{j_{m}}^{\prime} \in N \backslash\left\{j_{m}\right\}$, suppose that $\left\{i_{1}, \ldots, i_{m}\right\} \subset \varphi^{*}(x), x_{j_{1}}, \ldots, x_{j_{m}} \notin\left\{i_{1}, \ldots, i_{m}\right\}$, and $x_{j_{1}}^{\prime}=i_{1}, \ldots, x_{j_{m}}^{\prime}=i_{m}$. Let $x^{\prime}=\left(x_{\left\{j_{1}, \ldots, j_{m}\right\}}^{\prime}, x_{N \backslash\left\{j_{1}, \ldots, j_{m}\right\}}\right)$. We now distinguish three possible cases: (i) $\left|F_{x}\right|>1$; (ii) $\left|F_{x}\right|=1$ and $m=1$; (iii) $\left|F_{x}\right|=1$ and $m>1$. If $\left|F_{x}\right|>1$, we have $\left\{i_{1}, \ldots, i_{m}\right\} \subset F_{x}$. Then, we have $\left\{i_{1}, \ldots, i_{m}\right\} \subset F_{x^{\prime}}$. Therefore, $\left\{i_{1}, \ldots, i_{m}\right\} \subset \varphi^{*}\left(x^{\prime}\right)$. If $\left|F_{x}\right|=1$ and $m=1$, we have either $i_{1} \in F_{x}$ or $i_{1} \in S_{x}$ with $s_{F}(x)-s_{S}(x)=1$. Then, in either of the two cases, we have $i_{1} \in F_{x^{\prime}}$. Therefore, $\left\{i_{1}\right\} \subset \varphi^{*}\left(x^{\prime}\right)$. If $\left|F_{x}\right|=1$ and $m>1$, we have $s_{F}(x)-s_{S}(x)=1$. Without loss of generality, assume that $i_{2}, \ldots, i_{m} \in S_{x}$, so we have $x_{i_{2}}, \ldots, x_{i_{m}} \in F_{x}$. If $i_{1} \in F_{x}$, then, we have $F_{x^{\prime}}=\left\{i_{1}\right\}, i_{2}, \ldots, i_{m} \in S_{x^{\prime}}$, and $s_{F}\left(x^{\prime}\right)-s_{S}\left(x^{\prime}\right)=1$. Moreover, since

\footnotetext{
${ }^{4} \mathrm{~A}$ formal definition of $F_{x_{-i}}$ will be as follows. For each $x \in N_{-}^{N}$, each $i \in N$, and each $j \in N$, define $s_{j}\left(x_{-i}\right)=\left|\left\{k \in N \backslash\{i, j\}: x_{k}=j\right\}\right|$, and then, define $F_{x_{-i}}=\left\{j \in N: s_{j}\left(x_{-i}\right)=\max _{k \in N} s_{k}\left(x_{-i}\right)\right\}$. Notice that $s_{i}\left(x_{-i}\right)=s_{i}(x)$ for any $x \in N_{-}^{N}$ and any $i \in N$.
} 
$x_{j_{1}}, \ldots, x_{j_{m}} \notin\left\{i_{1}\right\}$ and $x_{i_{2}}=\ldots=x_{i_{m}}=i_{1}$, we have $i_{2}, \ldots, i_{m} \notin\left\{j_{1}, \ldots, j_{m}\right\}$, implying that $x_{i_{2}}^{\prime}=\ldots=x_{i_{m}}^{\prime}=i_{1}$. Hence, we obtain $\left\{i_{1}, \ldots, i_{m}\right\} \subset \varphi^{*}\left(x^{\prime}\right)$. If $i_{1} \in S_{x}$, then, since $s_{F}(x)-s_{S}(x)=1$, we have $\left\{i_{1}, \ldots, i_{m}\right\} \subset F_{x^{\prime}}$. Therefore, we obtain $\left\{i_{1}, \ldots, i_{m}\right\} \subset \varphi^{*}\left(x^{\prime}\right)$.

We now turn to prove that $\varphi^{*}$ is the only minimal nomination rule satisfying the four axioms. Note that this is equivalent to proving that no other nomination rule satisfying the four axioms is minimal, which is, after all, equivalent to showing that, for any $\varphi \in \Phi$, we have $\varphi^{*}(x) \subset \varphi(x)$ for all $x \in N_{-}^{N}$. Here we prove the last statement in two steps: we first show that $F_{x} \subset \varphi(x)$ for all $x \in N_{-}^{N}$; and second show that $\left\{i \in S_{x}: x_{i} \in F_{x}\right\} \subset \varphi(x)$ whenever $x \in N_{-}^{N}$ is such that $\left|F_{x}\right|=1$ and $s_{F}(x)-s_{S}(x)=1$.

Step 1. $F_{x} \subset \varphi(x)$ for all $x \in N_{-}^{N}$.

This is shown by induction on $s_{F}(x)$, the first highest score in $x \in N_{-}^{N}$. First, let $x \in N_{-}^{N}$ be such that $s_{F}(x)=1$. Note that $F_{x}=N$. Now, suppose that, for the sake of contradiction, we have $i \notin \varphi(x)$ for some $i \in F_{x}$. Without loss of generality, assume that $1 \notin \varphi(x)$ and $x_{1}=2$. Consider $x^{\prime} \in N_{-}^{N}$ such that $x_{j}^{\prime}=j+1$ for all $j=1, \ldots, n-1$ and $x_{n}^{\prime}=1$. Then, since $s_{j}\left(x^{\prime}\right)=s_{j}(x)=1$ for all $j \in N$ and $x_{1}^{\prime}=x_{1}$, anonymity implies that $1 \notin \varphi\left(x^{\prime}\right)$. Consider $\pi \in \Pi_{N}$ such that $\pi(j)=x_{j}^{\prime}$ for all $j \in N$. Note that $\left(x^{\prime}\right)^{\pi}=x^{\prime}$. Then, symmetry implies that $\left[1 \notin \varphi\left(x^{\prime}\right) \Rightarrow 2 \notin \varphi\left(x^{\prime}\right)\right],\left[2 \notin \varphi\left(x^{\prime}\right) \Rightarrow 3 \notin \varphi\left(x^{\prime}\right)\right], \ldots$, and $\left[n-1 \notin \varphi\left(x^{\prime}\right) \Rightarrow n \notin \varphi\left(x^{\prime}\right)\right]$. Thus, we have $\varphi\left(x^{\prime}\right)=\emptyset$, a contradiction.

Next, for all $r=2, \ldots, n-1$, assume that $F_{x} \subset \varphi(x)$ whenever $x \in N_{-}^{N}$ is such that $s_{F}(x)=r-1$ (induction hypothesis). Let $x \in N_{-}^{N}$ be such that $s_{F}(x)=r$. We show that $F_{x} \subset \varphi(x)$. Suppose that $\left|F_{x}\right|=m$ and $F_{x}=\left\{i_{1}, \ldots, i_{m}\right\}$, where $1 \leq m \leq n / 2$. Let $H_{x}=\left\{h \in N: s_{h}(x)=0\right\}$ denote the set of people not obtaining any nomination from others in $x$. Note that, since $s_{F}(x)=r \geq 2$, we have $\left|H_{x}\right| \geq m$. Now, suppose that there exist $j_{1}, \ldots, j_{m} \in N$ and $h_{1}, \ldots, h_{m} \in H_{x}$ such that $x_{j_{1}}=i_{1}, \ldots, x_{j_{m}}=i_{m}$ and $j_{1} \neq h_{1}, \ldots, j_{m} \neq h_{m}$. Consider $x^{\prime} \in N_{-}^{N}$ such that $x_{j_{1}}^{\prime}=h_{1}, \ldots, x_{j_{m}}^{\prime}=h_{m}$ and $x_{i}^{\prime}=x_{i}$ for all $i \in N \backslash\left\{j_{1}, \ldots, j_{m}\right\}$. Then, we have $\left\{i_{1}, \ldots, i_{m}\right\} \subset F_{x^{\prime}}$ and $s_{F}\left(x^{\prime}\right)=r-1$. Therefore, induction hypothesis implies that $\left\{i_{1}, \ldots, i_{m}\right\} \subset \varphi\left(x^{\prime}\right)$. Hence, by monotonicity, we obtain $F_{x}=\left\{i_{1}, \ldots, i_{m}\right\} \subset \varphi(x)$. It remains to check that there always exist such $j_{1}, \ldots, j_{m} \in N$ and $h_{1}, \ldots, h_{m} \in H_{x}$. We distinguish two possible cases, namely, $\left|H_{x}\right|=1$ and $\left|H_{x}\right|>1$.

Case 1. $\left|H_{x}\right|=1$.

Since $\left|H_{x}\right| \geq m$, we have $m=1$. Let $h_{1} \in H_{x}$. Since $s_{i_{1}}(x)=r \geq 2$, there must be $j_{1} \in N$ such that $x_{j_{1}}=i_{1}$ and $j_{1} \neq h_{1}$.

Case 2. $\left|H_{x}\right|>1$.

Since $s_{F}(x)=r \geq 2>0$, there exist $j_{1}, \ldots, j_{m} \in N$ such that $x_{j_{1}}=i_{1}, \ldots, x_{j_{m}}=i_{m}$. We now choose $h_{1}, \ldots, h_{m} \in H_{x}$ so that $j_{1} \neq h_{1}, \ldots, j_{m} \neq h_{m}$. If $m=1$, since $\left|H_{x}\right|>1$, 
there must be $h_{1} \in H_{x}$ such that $j_{1} \neq h_{1}$. If $m>1$, we further distinguish three subcases with respect to the number of members in $\left\{j_{1}, \ldots, j_{m}\right\} \cap H_{x}$. Let $\left|\left\{j_{1}, \ldots, j_{m}\right\} \cap H_{x}\right|=l$. If $l=0$, no difficulty arises in our choice problem; for any way of choosing $h_{1}, \ldots, h_{m} \in$ $H_{x}$, we obtain $j_{1} \neq h_{1}, \ldots, j_{m} \neq h_{m}$. If $l \geq 1$, without loss of generality, assume that $j_{1}, \ldots, j_{l} \in\left\{j_{1}, \ldots, j_{m}\right\} \cap H_{x}$. If $l=1$, let $h_{m}=j_{1}$, and choose $h_{1}, \ldots, h_{m-1}$ arbitrarily from among $H_{x} \backslash\left\{h_{m}\right\}$. Then, since $m>1$, we obtain $j_{1} \neq h_{1}, \ldots, j_{m} \neq h_{m}$. If $l>1$, let $h_{1}=j_{2}, \ldots, h_{l-1}=j_{l}$, and $h_{l}=j_{1}$, and choose $h_{l+1}, \ldots, h_{m}$ (if $l<m$ ) arbitrarily from among $H_{x} \backslash\left\{h_{1}, \ldots, h_{l}\right\}$. Then, we obtain $j_{1} \neq h_{1}, \ldots, j_{m} \neq h_{m}$.

Step 2. $\left\{i \in S_{x}: x_{i} \in F_{x}\right\} \subset \varphi(x)$ if $\left|F_{x}\right|=1$ and $s_{F}(x)-s_{S}(x)=1$.

Let $x \in N_{-}^{N}$ be such that $\left|F_{x}\right|=1$ and $s_{F}(x)-s_{S}(x)=1$. Let $i \in S_{x}$ with $x_{i} \in F_{x}$. Note that, since $\left|F_{x}\right|=1$, we have $s_{F}(x) \geq 2$. Then, there exists $h \in N \backslash\left(F_{x} \cup S_{x}\right)$ such that $s_{h}(x)=0$. Let $x_{i}^{\prime}=h$. Then, since $\left|F_{x}\right|=1, i \in S_{x}, s_{F}(x)-s_{S}(x)=1$, and $x_{i} \in F_{x}$, we have $i \in F_{\left(x_{i}^{\prime}, x_{-i}\right)}$. Therefore, by Step 1, we have $i \in F_{\left(x_{i}^{\prime}, x_{-i}\right)} \subset \varphi\left(x_{i}^{\prime}, x_{-i}\right)$. Hence, by impartiality, we obtain $i \in \varphi(x)$.

We check that the four axioms in Theorem 1 are needed for its statement. We show that, if we drop each of the four axioms, there exists another nomination rule $\varphi$ that satisfies all the other axioms and that $\varphi^{*}(x) \not \subset \varphi(x)$ for some $x \in N_{-}^{N}$. All the verifications we omit here are established in Appendix.

Example 1 (Dropping impartiality). The plurality correspondence, defined by $\varphi(x)=F_{x}$ for all $x \in N_{-}^{N}$, satisfies anonymity, symmetry, and monotonicity, but not impartiality.

Example 2 (Dropping anonymity). Suppose $n \geq 4$. Consider the following subcorrespondence $\varphi$ of the plurality with runners-up: for all $x \in N_{-}^{N}$,

Case A. if $\left|F_{x}\right|>1$, and

(i) if $s_{F}(x)>1$, then $\varphi(x)=F_{x}$;

(ii) if $s_{F}(x)=1$ and $\mid\left\{i \in F_{x}: \exists i^{\prime} \in N \backslash\{i\}, x_{i^{\prime}}=i\right.$ and $\left.x_{i}=i^{\prime}\right\} \mid=n$, then $\varphi(x)=F_{x}$;

(iii) if $s_{F}(x)=1$ and $\mid\left\{i \in F_{x}: \exists i^{\prime} \in N \backslash\{i\}, x_{i^{\prime}}=i\right.$ and $\left.x_{i}=i^{\prime}\right\} \mid<n$, then $\varphi(x)=F_{x}^{*}$, where $F_{x}^{*}=\left\{i \in F_{x}: \exists j, k \in N \backslash\{i\}, x_{j}=i\right.$ and $\left.x_{k}=j\right\}$;

Case B. if $\left|F_{x}\right|=1, s_{F}(x)-s_{S}(x)=1$, and

(i) if $s_{F}(x)>2$, then $\varphi(x)=F_{x} \cup\left\{i \in S_{x}: x_{i} \in F_{x}\right\}$;

(ii) if $s_{F}(x)=2$ and $\mid\left\{i \in S_{x}: \exists i^{\prime} \in N \backslash\{i\}, x_{i^{\prime}}=i\right.$ and $\left.x_{i}=i^{\prime}\right\} \mid=n-3$, then $\varphi(x)=F_{x} \cup\left\{i \in S_{x}: x_{i} \in F_{x}\right\}$;

(iii) if $s_{F}(x)=2$ and $\mid\left\{i \in S_{x}: \exists i^{\prime} \in N \backslash\{i\}, x_{i^{\prime}}=i\right.$ and $\left.x_{i}=i^{\prime}\right\} \mid<n-3$, 
then $\varphi(x)=F_{x} \cup\left\{i \in S_{x}^{*}: x_{i} \in F_{x}\right\}$,

where $S_{x}^{*}=\left\{i \in S_{x}: \exists j, k \in N \backslash\{i\}, x_{j}=i\right.$ and $\left.x_{k}=j\right\}$;

Case C. if $\left|F_{x}\right|=1$ and $s_{F}(x)-s_{S}(x)>1$, then $\varphi(x)=F_{x}$.

This subcorrespondence satisfies impartiality, symmetry, and monotonicity, but not anonymity if $n \geq 5$.

Example 3 (Dropping symmetry). We introduce a subcorrespondence $\varphi$ of the plurality with runners-up mentioned in Tamura and Ohseto (2014). Fix an order on $N$. For any $x \in N_{-}^{N}$, the person $i$ being the first member of $F_{x}$ always wins, and there are two special cases in which there is one additional winner $j \neq i$ : (i) if $\left|F_{x}\right|>1, i \in F_{x}$, and $j$ is the second member of $F_{x}$ with $x_{j}=i$, then $\varphi(x)=\{i, j\}$; (ii) if $\left|F_{x}\right|=1, i \in F_{x}$, and $j$ is the first member of $S_{x}$ with $s_{i}(x)-s_{j}(x)=1, x_{j}=i$, and $j$ precedes $i$, then $\varphi(x)=\{i, j\}$. This subcorrespondence satisfies impartiality, anonymity, and monotonicity, but not symmetry.

Example 4 (Dropping monotonicity). Let $\varphi$ be such that, for all $x \in N_{-}^{N}$,

(a) if $s_{F}(x)=n-1$, then $\varphi(x)=\left\{i \in N: s_{i}(x)=1\right\}$;

(b) else, $\varphi(x)=\left\{i \in N: s_{i}(x) \geq 1\right\}$.

This nomination rule satisfies impartiality, anonymity, and symmetry, but not monotonicity.

We have seen in Example 2 that anonymity is necessary for Theorem 1 whenever $n \geq 5$. This is, however, no longer true if $n \leq 4$ since, in that case, we can establish the result without assuming anonymity. To see this, it suffices to show that symmetry implies that

$\varphi(x)=F_{x}=N$ whenever $s_{F}(x)=1$. Let $x \in N_{-}^{N}$ be such that $s_{F}(x)=1$. First, consider the case of $n=3$. Then, there always exist $i_{1}, i_{2}, i_{3} \in N$ such that $x_{i_{1}}=i_{2}, x_{i_{2}}=i_{3}$, and $x_{i_{3}}=i_{1}$. Consider $\pi \in \Pi_{N}$ such that $\pi(i)=x_{i}$. Then, since $x^{\pi}=x$, symmetry implies that $\varphi(x)=\left\{i_{1}, i_{2}, i_{3}\right\}$. Second, consider the case of $n=4$. In this case, we distinguish the following two subcases: (i) $\exists i_{1}, i_{2}, i_{3}, i_{4} \in N$ such that $x_{i_{1}}=i_{2}, x_{i_{2}}=i_{3}, x_{i_{3}}=i_{4}$, and $x_{i_{4}}=i_{1}$; (ii) $\exists i_{1}, i_{2}, j_{1}, j_{2} \in N$ such that $x_{i_{1}}=i_{2}, x_{i_{2}}=i_{1}, x_{j_{1}}=j_{2}$, and $x_{j_{2}}=j_{1}$. If (i), then by the same argument as with the case of $n=3$, we obtain $\varphi(x)=\left\{i_{1}, i_{2}, i_{3}, i_{4}\right\}$. If (ii), consider $\pi \in \Pi_{N}$ such that $\pi\left(i_{1}\right)=i_{2}, \pi\left(i_{2}\right)=i_{1}, \pi\left(j_{1}\right)=j_{2}$, and $\pi\left(j_{2}\right)=j_{1}$. Then, we have $x^{\pi}=x$. Therefore, symmetry implies that we have $\left\{i_{1}, i_{2}\right\} \subset \varphi(x)$ or $\left\{j_{1}, j_{2}\right\} \subset \varphi(x)$ (or both). On the other hand, consider $\pi^{\prime} \in \Pi_{N}$ such that $\pi^{\prime}\left(i_{1}\right)=j_{1}, \pi^{\prime}\left(i_{2}\right)=j_{2}$, $\pi^{\prime}\left(j_{1}\right)=i_{1}$, and $\pi^{\prime}\left(j_{2}\right)=i_{2}$. Then, we have $x^{\pi^{\prime}}=x$. Therefore, symmetry implies that we have $\left\{i_{1}, j_{1}\right\} \subset \varphi(x)$ or $\left\{i_{2}, j_{2}\right\} \subset \varphi(x)$ (or both). Hence, we obtain $\varphi(x)=\left\{i_{1}, i_{2}, j_{1}, j_{2}\right\}$.

\section{Concluding remarks}

We showed that plurality with runners-up is the only minimal nomination rule satisfying impartiality, anonymity, symmetry, and monotonicity. It would be fair to say that our 
three axioms, as well as impartiality, are desirable properties in practical situations. Then, our result suggests that plurality with runners-up is a reasonable impartial nomination rule to use in such situations. Moreover, as we have seen in the proof of Theorem 1, the rule becomes simple enough for practical use if it is represented as follows: a person wins if and only if she is one of the plurality winners when her nomination is omitted.

Given our result, one may wonder if one can establish a complete characterization of the plurality with runners-up, that is, whether one can find a set of axioms that deduces the rule. In regard to this, we currently know that, if $n=4$, then plurality with runners-up is the unique nomination rule satisfying impartiality, symmetry, and positive and negative unanimity mentioned in the introduction. ${ }^{5}$ However, we have not seen any such characterization if $n \geq 5$, so we leave this topic for future research.

\section{References}

de Clippel, G., Moulin, H., Tideman, N., 2008. Impartial division of a dollar. Journal of Economic Theory 139, 176-191.

Holzman, R., Moulin, H., 2013. Impartial nominations for a prize. Econometrica 81, 173196.

Tamura, S., Ohseto, S., 2014. Impartial nomination correspondences. Social Choice and Welfare 43, 47-54.

\footnotetext{
${ }^{5}$ The proof is available from the author.
} 


\section{Appendix}

In this appendix, we verify that each of the four examples introduced above actually satisfies the corresponding three axioms but not the one, as stated there.

Example 1 Let $\varphi$ be as in Example 1. We show that $\varphi$ satisfies anonymity, symmetry, and monotonicity, but not impartiality.

Anonymity Obvious.

Symmetry Obvious.

Monotonicity Obvious.

Non-impartiality Consider a nomination profile $x \in N_{-}^{N}$ such that $s_{F}(x)=1$. Then, we have $\varphi(x)=N$. Now, let $x_{i}^{\prime} \neq x_{i}$ for some $i \in N$. Then, we have $F_{\left(x_{i}^{\prime}, x_{-i}\right)}=\left\{x_{i}^{\prime}\right\}$. Therefore, we have $i \notin \varphi\left(x_{i}^{\prime}, x_{-i}\right)$, violating impartiality.

Example 2 Let $\varphi$ be as in Example 2. We show that $\varphi$ satisfies impartiality, symmetry, monotonicity, but not anonymity if $n \geq 5$.

Impartiality For all $x \in N_{-}^{N}$ and all $i \in N$, suppose that $i \in \varphi(x)$. Let $x_{i}^{\prime} \neq x_{i}$. For simplicity of notation, let $x^{\prime}=\left(x_{i}^{\prime}, x_{-i}\right)$. Now, we distinguish three cases of $\mathrm{A}, \mathrm{B}$, and $\mathrm{C}$, as in the definition of $\varphi$.

Case A. $\left|F_{x}\right|>1$.

Then, we have $i \in F_{x}$. We further distinguish three subcases as in the definition of $\varphi$.

(i) $s_{F}(x)>1$. If $x_{i}^{\prime} \in F_{x} \backslash\{i\}$, then $i \in S_{x^{\prime}}, F_{x^{\prime}}=\left\{x_{i}^{\prime}\right\}, s_{F}\left(x^{\prime}\right)-s_{S}\left(x^{\prime}\right)=1$, and $s_{F}\left(x^{\prime}\right)>2$. Therefore, we obtain $i \in \varphi\left(x^{\prime}\right)$. If $x_{i}^{\prime} \notin F_{x}$, then $i \in F_{x^{\prime}}$, implying that $s_{F}\left(x^{\prime}\right)=s_{F}(x)>1$. Therefore, $i \in \varphi\left(x^{\prime}\right)$.

(ii) $s_{F}(x)=1$ and $\mid\left\{i \in F_{x}: \exists i^{\prime} \in N \backslash\{i\}, x_{i^{\prime}}=i\right.$ and $\left.x_{i}=i^{\prime}\right\} \mid=n$. Then, there (uniquely) exists $i^{\prime} \in N \backslash\{i\}$ such that $x_{i^{\prime}}=i$ and $x_{i}=i^{\prime}$. Suppose $x_{i}^{\prime}=j$ for some $j \in N \backslash\left\{i, i^{\prime}\right\}$. Note that, since $\mid\left\{i \in F_{x}: \exists i^{\prime} \in N \backslash\{i\}, x_{i^{\prime}}=i\right.$ and $\left.x_{i}=i^{\prime}\right\} \mid=n$ and $x_{i}=i^{\prime} \neq j$, we have $x_{j} \neq i, i^{\prime}$. Now, since $s_{F}(x)=1$, we have $i \in S_{x^{\prime}}, F_{x^{\prime}}=\left\{x_{i}^{\prime}\right\}=\{j\}, s_{F}\left(x^{\prime}\right)-s_{S}\left(x^{\prime}\right)=1$, and $s_{F}\left(x^{\prime}\right)=s_{j}\left(x^{\prime}\right)=2$. Moreover, since $\mid\left\{i \in F_{x}: \exists i^{\prime} \in N \backslash\{i\}, x_{i^{\prime}}=i\right.$ and $\left.x_{i}=i^{\prime}\right\} \mid=n$, $S_{x^{\prime}}=F_{x} \backslash\left\{i^{\prime}, j\right\}=N \backslash\left\{i^{\prime}, j\right\}, x_{i}^{\prime}=j$, and $x_{j}^{\prime}=x_{j} \neq i, i^{\prime}$, we have $\mid\left\{i \in S_{x^{\prime}}: \exists i^{\prime} \in\right.$ $N \backslash\{i\}, x_{i^{\prime}}^{\prime}=i$ and $\left.x_{i}^{\prime}=i^{\prime}\right\} \mid=n-3$. Therefore, we obtain $i \in \varphi\left(x^{\prime}\right)$.

(iii) $s_{F}(x)=1$ and $\mid\left\{i \in F_{x}: \exists i^{\prime} \in N \backslash\{i\}, x_{i^{\prime}}=i\right.$ and $\left.x_{i}=i^{\prime}\right\} \mid<n$. Then, there exist $j, k \in N \backslash\{i\}$ such that $x_{j}=i$ and $x_{k}=j$. Now, for any $x_{i}^{\prime} \neq x_{i}$, since $s_{F}(x)=1$, we have $i \in S_{x^{\prime}}, F_{x^{\prime}}=\left\{x_{i}^{\prime}\right\}, s_{F}\left(x^{\prime}\right)-s_{S}\left(x^{\prime}\right)=1$, and $s_{F}\left(x^{\prime}\right)=2$. Therefore, since $x_{j}^{\prime}=x_{j}=i$ and $x_{k}^{\prime}=x_{k}=j$, regardless of $\mid\left\{i \in S_{x^{\prime}}: \exists i^{\prime} \in N \backslash\{i\}, x_{i^{\prime}}^{\prime}=i\right.$ and $\left.x_{i}^{\prime}=i^{\prime}\right\} \mid$, we obtain $i \in \varphi\left(x^{\prime}\right)$. 
Case B. $\left|F_{x}\right|=1$ and $s_{F}(x)-s_{S}(x)=1$.

First, consider the case that $i \in F_{x}$. Note that, since $\left|F_{x}\right|=1$, we have $s_{F}(x) \geq 2$. Now, for any $x_{i}^{\prime} \neq x_{i}$, since $F_{x}=\{i\}$, we have $i \in F_{x^{\prime}}$. Therefore, $s_{F}\left(x^{\prime}\right)=s_{F}(x) \geq 2$. Hence, regardless of $\left|F_{x^{\prime}}\right|$, we obtain $i \in \varphi\left(x^{\prime}\right)$.

Next, consider the case that $i \in S_{x}$. Then, we have $x_{i} \in F_{x}$. Now, we distinguish three subcases as in the definition of $\varphi$.

(i) $s_{F}(x)>2$. Since $F_{x}=\left\{x_{i}\right\}$ and $s_{F}(x)-s_{S}(x)=1$, we have either $i \in F_{x^{\prime}}$ (if $x_{i}^{\prime} \notin S_{x}$ ) or $i \in S_{x^{\prime}}$ with $s_{F}\left(x^{\prime}\right)-s_{S}\left(x^{\prime}\right)=1$ (if $x_{i}^{\prime} \in S_{x} \backslash\{i\}$ ). If $i \in F_{x^{\prime}}$, then, since $s_{F}(x)>2$, we have $s_{F}\left(x^{\prime}\right) \geq 2$. Therefore, regardless of $\left|F_{x^{\prime}}\right|$, we obtain $i \in \varphi\left(x^{\prime}\right)$. If $i \in S_{x^{\prime}}$, then, since $F_{x^{\prime}}=\left\{x_{i}^{\prime}\right\}, x_{i}^{\prime} \in S_{x} \backslash\{i\}$, and $s_{F}(x)-s_{S}(x)=1$, we have $s_{F}\left(x^{\prime}\right)=s_{F}(x)>2$. Therefore, we obtain $i \in \varphi\left(x^{\prime}\right)$.

(ii) $s_{F}(x)=2$ and $\mid\left\{i \in S_{x}: \exists i^{\prime} \in N \backslash\{i\}, x_{i^{\prime}}=i\right.$ and $\left.x_{i}=i^{\prime}\right\} \mid=n-3$. Suppose $F_{x}=\{j\}$ for some $j \in N \backslash\{i\}$. Note that, since $s_{F}(x)=s_{j}(x)=2$ and $x_{i}=j$, there uniquely exists $k \in N \backslash\{i, j\}$ such that $x_{k}=j$. Note also that we have the following two cases: (a) $i \in F_{x^{\prime}}$ with $s_{F}\left(x^{\prime}\right)=1$ (if $x_{i}^{\prime} \notin S_{x}$, namely, $x_{i}^{\prime}=h$ for unique $h \in N \backslash\{i\}$ such that $s_{h}(x)=0$ ); (b) $i \in S_{x^{\prime}}$ with $F_{x^{\prime}}=\left\{x_{i}^{\prime}\right\}, s_{F}\left(x^{\prime}\right)-s_{S}\left(x^{\prime}\right)=1$, and $s_{F}\left(x^{\prime}\right)=2$ (if $\left.x_{i}^{\prime} \in S_{x} \backslash\{i\}\right)$. Then, if $x_{j}=i$, since $x_{k}=j$, we obtain $i \in \varphi\left(x^{\prime}\right)$ in either of the two cases. If $x_{j} \neq i$, then, since $x_{i}=j, \mid\left\{i \in S_{x}: \exists i^{\prime} \in N \backslash\{i\}, x_{i^{\prime}}=i\right.$ and $\left.x_{i}=i^{\prime}\right\} \mid=n-3$, and $S_{x}=N \backslash\{h, j\}$, we have $\left\{i \in S_{x}: \exists i^{\prime} \in N \backslash\{i\}, x_{i^{\prime}}=i\right.$ and $\left.x_{i}=i^{\prime}\right\}=S_{x} \backslash\{i\}$, which, since $s_{S}(x)=1$, implies that $x_{h}=i$, and thus, $k \in S_{x}$ and $x_{j}=k$ (remember $x_{k}=j$ ). Therefore, if (b), since $\mid\left\{i \in S_{x}\right.$ : $\exists i^{\prime} \in N \backslash\{i\}, x_{i^{\prime}}=i$ and $\left.x_{i}=i^{\prime}\right\} \mid=n-3, S_{x^{\prime}}=\left(S_{x} \backslash\left\{x_{i}^{\prime}\right\}\right) \cup\{j\}, x_{k}=j$, and $x_{j}=k$, we have $\mid\left\{i \in S_{x^{\prime}}: \exists i^{\prime} \in N \backslash\{i\}, x_{i^{\prime}}^{\prime}=i\right.$ and $\left.x_{i}^{\prime}=i^{\prime}\right\} \mid=n-3$. Hence, we obtain $i \in \varphi\left(x^{\prime}\right)$. If (a), since $\left\{i \in S_{x}: \exists i^{\prime} \in N \backslash\{i\}, x_{i^{\prime}}=i\right.$ and $\left.x_{i}=i^{\prime}\right\}=S_{x} \backslash\{i\}=N \backslash\{h, i, j\}, x_{i}^{\prime}=h$, $x_{h}=i, x_{j}=k$, and $x_{k}=j$, we have $\mid\left\{i \in F_{x^{\prime}}: \exists i^{\prime} \in N \backslash\{i\}, x_{i^{\prime}}^{\prime}=i\right.$ and $\left.x_{i}^{\prime}=i^{\prime}\right\} \mid=n$. Therefore, we obtain $i \in \varphi\left(x^{\prime}\right)$.

(iii) $s_{F}(x)=2$ and $\mid\left\{i \in S_{x}: \exists i^{\prime} \in N \backslash\{i\}, x_{i^{\prime}}=i\right.$ and $\left.x_{i}=i^{\prime}\right\} \mid<n-3$. Then, there exist $j, k \in N \backslash\{i\}$ such that $x_{j}=i$ and $x_{k}=j$. Now, since $F_{x}=\left\{x_{i}\right\}, s_{F}(x)-s_{S}(x)=1$, and $s_{F}(x)=2$, we have either $i \in F_{x^{\prime}}$ with $s_{F}\left(x^{\prime}\right)=1$ (if $x_{i}^{\prime} \notin S_{x}$ ) or $i \in S_{x^{\prime}}$ with $F_{x^{\prime}}=\left\{x_{i}^{\prime}\right\}$, $s_{F}\left(x^{\prime}\right)-s_{S}\left(x^{\prime}\right)=1$, and $s_{F}\left(x^{\prime}\right)=2$ (if $x_{i}^{\prime} \in S_{x} \backslash\{i\}$ ). Therefore, since $x_{j}^{\prime}=x_{j}=i$ and $x_{k}^{\prime}=x_{k}=j$, we obtain $i \in \varphi\left(x^{\prime}\right)$ in either of the two cases.

Case C. $\left|F_{x}\right|=1$ and $s_{F}(x)-s_{S}(x)>1$.

Then, we have $i \in F_{x}$. Note that, since $F_{x}=\{i\}$ and $s_{F}(x)-s_{S}(x)>1$, we have $F_{x^{\prime}}=\{i\}$ whatever $x_{i}^{\prime} \neq x_{i}$ is. Hence, we obtain $i \in \varphi\left(x^{\prime}\right)$.

Symmetry One can verify this samely as with the case for the plurality with runners-up if taking care of the following two simple facts: (i) for any $\pi \in \Pi_{N}$, any $x \in N_{-}^{N}$, and any $i, i^{\prime} \in N$, we have $x_{\pi\left(i^{\prime}\right)}^{\pi}=\pi(i)$ and $x_{\pi(i)}^{\pi}=\pi\left(i^{\prime}\right)$ whenever $x_{i^{\prime}}=i$ and $x_{i}=i^{\prime}$; (ii) for any 
$\pi \in \Pi_{N}$, any $x \in N_{-}^{N}$, and any $i, j, k \in N$, we have $x_{\pi(j)}^{\pi}=\pi(i)$ and $x_{\pi(k)}^{\pi}=\pi(j)$ whenever $x_{j}=i$ and $x_{k}=j$.

Monotonicity For all $x \in N_{-}^{N}$, all $i_{1}, \ldots, i_{m} \in N$, all $j_{1}, \ldots, j_{m} \in N$, all $x_{j_{1}}^{\prime} \in N \backslash\left\{j_{1}\right\}, \ldots$, and all $x_{j_{m}}^{\prime} \in N \backslash\left\{j_{m}\right\}$, suppose that $\left\{i_{1}, \ldots, i_{m}\right\} \subset \varphi^{*}(x), x_{j_{1}}, \ldots, x_{j_{m}} \notin\left\{i_{1}, \ldots, i_{m}\right\}$, and $x_{j_{1}}^{\prime}=i_{1}, \ldots, x_{j_{m}}^{\prime}=i_{m}$. Let $x^{\prime}=\left(x_{\left\{j_{1}, \ldots, j_{m}\right\}}^{\prime}, x_{N \backslash\left\{j_{1}, \ldots, j_{m}\right\}}\right)$. If $\left|F_{x}\right|>1$, we have $\left\{i_{1}, \ldots, i_{m}\right\} \subset F_{x}$, and thus $\left\{i_{1}, \ldots, i_{m}\right\} \subset F_{x^{\prime}}$. Moreover, since $s_{F}(x) \geq 1$, we have $s_{F}\left(x^{\prime}\right)>1$. Hence, we obtain $\left\{i_{1}, \ldots, i_{m}\right\} \subset \varphi^{*}\left(x^{\prime}\right)$. If $\left|F_{x}\right|=1$, note that $s_{F}(x) \geq 2$. Now, we distinguish two cases, namely, $m=1$ and $m>1$. If $m=1$, we have either $i_{1} \in F_{x}$ or $i_{1} \in S_{x}$ with $s_{F}(x)-s_{S}(x)=1$. Then, in either of the two cases, we have $i_{1} \in F_{x^{\prime}}$, which, since $s_{F}(x) \geq 2$, implies that $s_{F}\left(x^{\prime}\right) \geq 2$. Therefore, regardless of $\left|F_{x^{\prime}}\right|$, we obtain $i_{1} \in \varphi\left(x^{\prime}\right)$. If $m>1$, assume without loss of generality that $i_{2}, \ldots, i_{m} \in S_{x}$, so we have $x_{i_{2}}, \ldots, x_{i_{m}} \in F_{x}$. If $i_{1} \in F_{x}$, then, we have $F_{x^{\prime}}=\left\{i_{1}\right\}, i_{2}, \ldots, i_{m} \in S_{x^{\prime}}$, and $s_{F}\left(x^{\prime}\right)-s_{S}\left(x^{\prime}\right)=1$. Moreover, since $s_{F}(x) \geq 2$, we have $s_{F}\left(x^{\prime}\right)>2$. On the other hand, since $x_{i_{2}}=\ldots=x_{i_{m}}=i_{1}$, we have $x_{i_{2}}^{\prime}=\ldots=x_{i_{m}}^{\prime}=i_{1}$. Hence, we obtain $\left\{i_{1}, \ldots, i_{m}\right\} \subset \varphi^{*}\left(x^{\prime}\right)$. If $i_{1} \in S_{x}$, then, since $s_{F}(x)-s_{S}(x)=1$ and $s_{F}(x) \geq 2$, we have $\left\{i_{1}, \ldots, i_{m}\right\} \subset F_{x^{\prime}}$ and $s_{F}\left(x^{\prime}\right) \geq 2$. Therefore, we obtain $\left\{i_{1}, \ldots, i_{m}\right\} \subset \varphi^{*}\left(x^{\prime}\right)$.

Non-anonymity Let $n \geq 5$. Consider five distinct persons $i_{1}, i_{2}, i_{3}, j_{1}, j_{2} \in N$ and a nomination profile $x \in N_{-}^{N}$ such that $s_{F}(x)=1, x_{i_{1}}=i_{2}, x_{i_{2}}=i_{3}, x_{i_{3}}=i_{1}, x_{j_{1}}=j_{2}$, and $x_{j_{2}}=j_{1}$. Then, since $\mid\left\{i \in F_{x}: \exists i^{\prime} \in N \backslash\{i\}, x_{i^{\prime}}=i\right.$ and $\left.x_{i}=i^{\prime}\right\} \mid<n, x_{j_{2}}=j_{1}$, $x_{j_{1}}=j_{2}$, and $s_{j_{2}}(x)=1$, we have $j_{1} \notin \varphi(x)$. Let $x_{i_{3}}^{\prime}=x_{j_{2}}=j_{1}$ and $x_{j_{2}}^{\prime}=x_{i_{3}}=i_{1}$. Then, since $s_{F}\left(x_{\left\{i_{3}, j_{2}\right\}}^{\prime}, x_{N \backslash\left\{i_{3}, j_{2}\right\}}\right)=1, x_{i_{3}}^{\prime}=j_{1}$, and $x_{i_{2}}=i_{3}$, we have $j_{1} \in \varphi\left(x_{\left\{i_{3}, j_{2}\right\}}^{\prime}, x_{N \backslash\left\{i_{3}, j_{2}\right\}}\right)$, violating anonymity.

Example 3 Let $\varphi$ be as in Example 3. We show that $\varphi$ satisfies impartiality, anonymity, and monotonicity, but not symmetry.

Impartiality Tamura and Ohseto (2014) show that $\varphi$ satisfies impartiality.

Anonymity This is essentially the same with the case for the plurality with runners-up, and we omit the proof.

Monotonicity For all $x \in N_{-}^{N}$, all $i_{1}, \ldots, i_{m} \in N$, all $j_{1}, \ldots, j_{m} \in N$, all $x_{j_{1}}^{\prime} \in N \backslash\left\{j_{1}\right\}, \ldots$, and all $x_{j_{m}}^{\prime} \in N \backslash\left\{j_{m}\right\}$, suppose that $\left\{i_{1}, \ldots, i_{m}\right\} \subset \varphi^{*}(x), x_{j_{1}}, \ldots, x_{j_{m}} \notin\left\{i_{1}, \ldots, i_{m}\right\}$, and $x_{j_{1}}^{\prime}=i_{1}, \ldots, x_{j_{m}}^{\prime}=i_{m}$ (here $m=1$ or $m=2$ ). Let $x^{\prime}=\left(x_{\left\{j_{1}, \ldots, j_{m}\right\}}^{\prime}, x_{N \backslash\left\{j_{1}, \ldots, j_{m}\right\}}\right)$. First, consider the case that $\left|F_{x}\right|>1$. In this case, we have $\left\{i_{1}, \ldots, i_{m}\right\} \subset F_{x}$. If $m=1$, then we have $F_{x^{\prime}}=\left\{i_{1}\right\}$, and thus $\left\{i_{1}\right\} \subset \varphi\left(x^{\prime}\right)$. If $m=2$, assume without loss of generality that $i_{1}$ and $i_{2}$ are the first and the second members of $F_{x}$ with $x_{i_{2}}=i_{1}$, respectively. Then, we have $F_{x^{\prime}}=\left\{i_{1}, i_{2}\right\}$ with $i_{1}$ and $i_{2}$ being the first and the second members of $F_{x^{\prime}}$, respectively. Moreover, since $x_{j_{1}}, x_{j_{2}} \notin\left\{i_{1}\right\}$ and $x_{i_{2}}=i_{1}$, we have $i_{2} \notin\left\{j_{1}, j_{2}\right\}$, implying that $x_{i_{2}}^{\prime}=x_{i_{2}}=i_{1}$. Therefore, we obtain $\left\{i_{1}, i_{2}\right\} \subset \varphi\left(x^{\prime}\right)$. 
Second, consider the case that $\left|F_{x}\right|=1$. If $m=1$, we have either $i_{1} \in F_{x}$ or $i_{1} \in S_{x}$ with $s_{F}(x)-s_{S}(x)=1$. If the former, then we have $F_{x^{\prime}}=\left\{i_{1}\right\}$, and thus $\left\{i_{1}\right\} \subset \varphi\left(x^{\prime}\right)$. If the latter, $i_{1}$ is the first member of $S_{x}$ nominating the unique member of $F_{x}$, say, $k$, and $i_{1}$ precedes $k$. Now, since $F_{x}=\{k\}$ and $s_{F}(x)-s_{S}(x)=1$, we have either $F_{x^{\prime}}=\left\{i_{1}\right\}$ (if $x_{j_{1}}=k$ ) or $F_{x^{\prime}}=\left\{i_{1}, k\right\}$ with $i_{1}$ preceding $k$ (if $x_{j_{1}} \neq k$ ). Therefore, in either case, we obtain $\left\{i_{1}\right\} \subset \varphi\left(x^{\prime}\right)$. If $m=2$, assume without loss of generality that $F_{x}=\left\{i_{1}\right\}, i_{2} \in S_{x}$, $s_{i_{1}}(x)-s_{i_{2}}(x)=1, x_{i_{2}}=i_{1}$, and $i_{2}$ precedes $i_{1}$. Then, we have $F_{x^{\prime}}=\left\{i_{1}\right\}, S_{x^{\prime}}=\left\{i_{2}\right\}$, $s_{i_{1}}\left(x^{\prime}\right)-s_{i_{2}}\left(x^{\prime}\right)=1$, and $i_{2}$ precedes $i_{1}$. Moreover, since $x_{i_{2}}=i_{1}$, we have $x_{i_{2}}^{\prime}=i_{1}$. Hence, we obtain $\left\{i_{1}, i_{2}\right\} \subset \varphi\left(x^{\prime}\right)$.

Non-symmetry Obvious.

Example 4 Let $\varphi$ be as in Example 4. We show that $\varphi$ satisfies impartiality, anonymity, symmetry, but not monotonicity.

Impartiality For all $x \in N_{-}^{N}$ and all $i \in N$, suppose that $i \in \varphi(x)$. Let $x_{i}^{\prime} \neq x_{i}$. Note that $s_{i}\left(x_{i}^{\prime}, x_{-i}\right)=s_{i}(x)$. Then, if $s_{F}(x)=n-1$, we have $s_{i}(x)=1$, and thus $s_{i}\left(x_{i}^{\prime}, x_{-i}\right)=1$. Hence, we obtain $i \in \varphi\left(x_{i}^{\prime}, x_{-i}\right)$. If $s_{F}(x)<n-1$, we have $s_{i}(x)=1$ or $s_{i}(x)>1$. If $s_{i}(x)=1$, we have $s_{i}\left(x_{i}^{\prime}, x_{-i}\right)=1$, and thus $i \in \varphi\left(x_{i}^{\prime}, x_{-i}\right)$. If $s_{i}(x)>1$, we have $s_{i}\left(x_{i}^{\prime}, x_{-i}\right)=s_{i}(x)>1$. Therefore, whether $s_{i}(x)=s_{F}(x)$ or $s_{i}(x)<s_{F}(x)$, we have $s_{F}\left(x_{i}^{\prime}, x_{-i}\right)<n-1$. Hence, we obtain $i \in \varphi\left(x_{i}^{\prime}, x_{-i}\right)$.

Anonymity Obvious.

Symmetry Obvious.

Non-monotonicity Consider a nomination profile $x \in N_{-}^{N}$ such that $s_{i}(x)=n-2$ for some $i \in N$. Then, we have $\{i\} \subset \varphi(x)$. Note that, since $s_{i}(x)=n-2<n-1$, there exists $j \in N \backslash\{i\}$ such that $x_{j} \neq i$. Let $x_{j}^{\prime}=i$. Then, we have $s_{i}\left(x_{j}^{\prime}, x_{-j}\right)=n-1$. Therefore, we have $i \notin \varphi\left(x_{j}^{\prime}, x_{-j}\right)$, violating monotonicity. 